\title{
Aproximación a la concepción de la salud mental para los pueblos indígenas de Colombia
}

\author{
Approaching the concept of mental health for indigenous peoples \\ in Colombia
}

Eliana María Montoya Vélez (https://orcid.org/0000-0002-3358-380X) ${ }^{1}$ Jennifer Marcela López Ríos (https://orcid.org/0000-0003-3629-9377) ${ }^{1}$ Sergio Cristancho Marulanda (https://orcid.org/0000-0001-5402-7530) ${ }^{1}$ Marcela Cristina Valencia Franco (https://orcid.org/0000-0001-8837-9307) ${ }^{2}$ Oscar David Montero de La Rosa (https://orcid.org/0000-0003-3208-6833) ${ }^{3}$ Dora María Hernández Holguín (https://orcid.org/0000-0002-1050-6625) ${ }^{1}$
${ }^{1}$ Facultad Nacional de Salud Publica, Universidad de Antioquia. Calle 62 \#52-59. Medellín Antioquia Colômbia. eliana.montoya. velez@gmail.com

${ }^{2}$ Facultad de Medicina, Universidad CES. Medellin Colombia.

${ }^{3}$ Organización Nacional Indígena de Colombia. Bogotá Colombia.

\begin{abstract}
This study sought to perceive the concept of mental health from the perspective of $\mathrm{Co}$ lombian indigenous peoples. A qualitative survey from a historic-hermeneutical standpoint was conducted by means of interviews with a stratified intentional sample of 10 leaders with experience in indigenous mental health. After being duly transcribed, interview materials were analyzed using a grounded theory approach. Findings were structured around four analytical categories: mental health perspectives, elements, scenarios, and challenges. It was revealed that some indigenous people do not perceive mental health as an indigenous concept. Some perceive it from a morbicentric western perspective and others from an ancestral holistic standpoint. They describe positive elements (good living, spirituality, harmony with mother earth) and negative aspects (acculturation, discrimination, violence, disobedience). Mental health scenarios included the land itself and the health care system, in particular the Intercultural Indigenous Health System (SISPI). Challenges include the need to conduct more research on this topic, consolidating SISPI, and fostering ancestral knowledge.
\end{abstract}

Key words Mental health, Culture, Colombia, Indigenous peoples
Resumen Este estudio tuvo como objetivo comprender el concepto de salud mental desde la perspectiva de los pueblos indígenas de Colombia. Se realizó una investigación con enfoque cualitativo, de tipo histórico hermenéutico a través de entrevistas a una muestra intencional estratifcada de 10 líderes con experiencia en el tema de salud indígena. Luego de transcritas, se realizó el análisis de las entrevistas con herramientas de la teoría fundada. Los hallazgos se centraron en cuatro categorías analiticas: perspectivas de la salud mental, elementos, escenarios y retos. Se encontró que algunos indígenas no asumen la salud mental como un concepto propio. Entre los restantes, algunos lo asumen desde una perspectiva occidental morbicéntrica y los demás desde una perspectiva ancestral holistica. Se describen elementos positivos (buen vivir, espiritualidad, armonía con la madre tierra) y negativos (aculturación, discriminación, violencia, desobediencia). Los escenarios de la salud mental que mencionaron los participantes son el territorio y el sistema de salud, en particular el Sistema Indígena de Salud Propio e Intercultural- SISPI. Los retos incluyen la necesidad de generar más investigaciones en este tema, el avance en la consolidación del SISPI y el fortalecimiento de los saberes ancestrales.

Palabras clave Salud mental, Cultura, Colombia, Población indígena 


\section{Introducción y antecedentes}

La salud mental, es definida por la Organización Mundial de la Salud (OMS) como "un estado de bienestar en el cual el individuo es consciente de sus propias capacidades, puede afrontar las tensiones normales de la vida, puede trabajar de forma productiva y fructífera y es capaz de hacer una contribución a su comunidad"1. A pesar de lo amplia que parece ser esta definición, su operacionalización en planes y programas específicos de salud mental a nivel gubernamental e institucional se limita a aspectos puntuales como la gestión del riesgo ${ }^{2}$. Sin embargo, las concepciones acerca de la salud, incluida la mental, varían en función de la cultura ${ }^{3}$, por lo tanto esta visión morbicéntrica, fragmentada y basada en la oferta de servicios, no responde adecuadamente a la integralidad y complejidad de la respuesta requerida para disminuir las inequidades.

En contraste, para varios pueblos indígenas del mundo, la salud es entendida como buen vi$v i r$. Es un concepto integral que toma en cuenta aspectos físicos, mentales, sociales, espirituales y ambientales, relacionados con el fortalecimiento cultural, la autonomía alimentaria, la cosmovisión y la territorialidad, en relación con la naturaleza y la medicina tradicional ${ }^{4}$. Entre tanto, la salud mental, para algunos pueblos indígenas australianos, es definida como "la capacidad del individuo, los grupos y el medio ambiente de interactuar entre sí de una manera que promueva el bienestar subjetivo, el desarrollo óptimo y el uso de las habilidades mentales (cognitiva, afectiva y relacional), los logros de metas individuales y colectivas, que sean consistentes con el logro y la existencia de condiciones fundamentales de equidad" '. ¿Pero, como conciben la salud mental los indígenas que habitan el territorio colombiano?

Actualmente, habitan en el mundo aproximadamente 370 millones de indígenas, correspondiente al $5 \%$ de la población general. Se encuentran agrupados en alrededor 5.000 culturas que hablan entre 6.000 y 7.000 lenguas $^{6}$. Desde una perspectiva epidemiológica occidental, los principales problemas mentales que sufren son: consumo de sustancias psicoactivas, alcoholismo, depresión y suicidio ${ }^{1,7}$, asociados al intenso estrés social, traumas históricos, abuso sexual, duelos no resueltos, pérdida del territorio y del control sobre sus condiciones de vida, la supresión del sistema de creencias y la espiritualidad, el quiebre de sus instituciones políticas y sociales, y la discriminación racial ${ }^{8}$.
Los pueblos indígenas de Latinoamérica sufren alta vulnerabilidad social y acceso limitado a los servicios de salud mental, especialmente en áreas rurales. Resultado de procesos de exclusión social, discriminación, pobreza y acceso limitado a los servicios de salud 9 . Un factor que puede contribuir al aumento de las inequidades en salud mental es la falta de comprensión de los formuladores de política pública e implementadores de programas, acerca de cómo las poblaciones más afectadas la conciben. Este desconocimiento puede conllevar a una orientación no oportuna de los programas locales de promoción, prevención y atención alrededor de temas priorizados desde la perspectiva de los pueblos, descontextualizados culturalmente, inoportunos, e incluso nocivos en la preservación de valores, conocimientos y prácticas ancestrales.

En Colombia, factores como la violencia y el conflicto armado afectan en gran medida el bienestar de las comunidades, generando desplazamiento y marginación ${ }^{10}$. La vulnerabilidad que tienen estos pueblos frente al conflicto, y dinámicas de mercado impulsadas por el mismo Estado, ponen en amenaza su autonomía y derechos consagrados en la constitución y mandatos internacionales ${ }^{11}$. Uno de los principales problemas es el suicidio; mientras que en la población general colombiana la tasa es de 4.4 por cada 100.000 habitantes, en los indígenas es de 500 por cada 100.000 habitantes $^{12}$.

Con estos antecedentes, en 2006 un grupo de investigadores con apoyo de la Organización Panamericana de la Salud (OPS) realizó una revisión de la literatura (estado del arte) sobre la salud mental de los pueblos indígenas en el periodo comprendido entre los años de 1995 y $2005^{13}$. Dicho estudio aportó información para las instituciones relacionadas, pero también sugirió la necesidad de incorporar el concepto indígena de salud mental en futuros estudios de este tipo, así como en el sistema de salud, con el fin de facilitar el acceso y la cobertura de la atención a esta población ${ }^{10}$. De esta manera, el presente estudio se realiza en seguimiento al anterior. Este artículo muestra los resultados investigativos de la primera aproximación a la comprensión de las concepciones sobre la salud mental en varios pueblos indígenas de Colombia, como parte del estado del arte, en el cual se buscaba comprender el concepto de salud mental desde la perspectiva de los pueblos indígenas de Colombia. 
Se realizó un estudio cualitativo usando el enfoque histórico hermenéutico, según Habermas como una perspectiva que permite una comprensión profunda del fenómeno de interés en su propio contexto ${ }^{14}$. Se realizó una estrategia de muestreo intencional estratificado ${ }^{15}$ por macroregiones de la Organización Nacional Indígena de Colombia (ONIC) ${ }^{16}$, para lo cual se identificaron líderes representantes de diferentes regiones del país reconocidos por las organizaciones locales y comunidades a las cuales pertenecen, con experiencia en el tema de salud indígena. Se seleccionaron 10 participantes que cumplían con los siguientes criterios: ser indígena, en pleno uso de sus facultades, reconocido por la ONIC como líder, tener experiencia en el tema de salud y/o salud mental indígena, español-hablante, y con disponibilidad para atender la entrevista vía telefónica. Es de aclarar que uno de los participantes realizó la entrevista vía e-mail dada las limitaciones que se presentaron para contactarlo vía telefónica. Para éste último caso, lo que se hizo fue realizar el análisis de la misma y se compartió con el participante para validar la información e interpretación que se le dio a sus respuestas. Dicho participante validó la información y realizó las correcciones correspondientes. La muestra final de estos 10 líderes indígenas estuvo conformada por 4 mujeres y 6 hombres representantes de las 5 macro regiones de la ONIC (Cuadro 1).

Previo consentimiento informado verbal, se realizaron las entrevistas semi-estructuradas telefónicas utilizando una guía de preguntas. Las entrevistas se orientaron a indagar sobre los significados de salud mental en los pueblos indígenas, así como palabras relacionadas con la misma, y posibles fuentes de información al respecto. Las entrevistas fueron grabadas, transcritas verba- tim. A cada transcripción se les asignó un código de identificación para preservar la identidad del participante.

Para el análisis se utilizaron herramientas de la Teoría Fundada ${ }^{17}$. Se realizó una matriz de codificación en hoja de cálculo Excel $2013^{\circledR}$ con la intención de tener un acercamiento inicial a la información. Posterior a ello se realizó un proceso de codificación abierta con base en lo cual se agruparon los códigos similares a través de un proceso de codificación axial. Se exportaron los resultados de la codificación abierta y axial a Atlas Ti $6.2{ }^{\circledR}$, etiquetando los textos mediante las subcategorías y categorías identificadas en la codificación axial. Finalmente se realizó un mapa conceptual para sintetizar la información y facilitar la visualización de los hallazgos.

Los métodos e instrumentos utilizados en este proyecto fueron aprobados por el Comité de Ética de la institución académica que lideró el proyecto.

\section{Resultados}

Como resultado del proceso inicial de análisis en torno a la pregunta ¿qué es la salud mental para su comunidad? se obtuvieron 160 códigos abiertos, los cuales se agruparon en 12 categorías descriptivas, que se agruparon en categorías analíticas: (1) perspectivas acerca de la salud mental, (2) elementos de la salud mental, (3) escenarios de la salud mental, y (4) retos para la salud mental indígena (Cuadro 2).

\section{Perspectivas acerca de la salud mental}

A este respecto, algunos entrevistados manifestaron que la salud mental es un concepto inexistente en la tradición de los Pueblos Indígenas:

Cuadro 1. Participantes del estudio, características demográficas.

\begin{tabular}{|l|l|l|l|}
\hline \multicolumn{1}{|c|}{ Sexo } & \multicolumn{1}{c|}{ Pueblo Indígena } & Macro Región según ONIC & \multicolumn{1}{c|}{ Departamento } \\
\hline Masculino & Uitoto & Amazonía & Amazonas \\
\hline Femenino & Zenú & Norte & Córdoba \\
\hline Masculino & Emberá-Chamí & Occidente & Caldas \\
\hline Masculino & Wayúu & Norte & La Guajira \\
\hline Femenino & Wiwa & Norte & Cesar \\
\hline Masculino & Pijao & Centro Oriente & Tolima \\
\hline Masculino & Cubeo & Orinoquía & Guainía \\
\hline Masculino & Tikuna & Amazonía & Amazonas \\
\hline Femenino & Nasa & Occidente & Cauca \\
\hline Femenino & Misak & Occidente & Cauca \\
\hline
\end{tabular}


Cuadro 2. Categorías descriptivas y analíticas.

\begin{tabular}{|l|l|}
\hline \multicolumn{1}{|c|}{ Categorías analíticas } & \multicolumn{1}{c|}{ Categorías descriptivas } \\
\hline \multirow{2}{*}{ (1) perspectivas acerca de la salud mental } & Abordaje de la salud mental desde la ancestralidad. \\
\cline { 2 - 2 } & Abordaje de la salud mental desde lo occidental. \\
\hline \multirow{5}{*}{ (2) elementos de la salud mental } & Concepción de la salud mental \\
\cline { 2 - 2 } & Causas de la salud mental \\
\cline { 2 - 2 } & Consecuencias de la salud mental \\
\cline { 2 - 2 } & $\begin{array}{l}\text { Percepciones del consumo de sustancias psicoactivas y } \\
\text { problemáticas en salud mental. }\end{array}$ \\
\hline \multirow{2}{*}{ (3) escenarios de la salud mental } & Avances en sistema de salud \\
\cline { 2 - 2 } & Avances del abordaje de salud mental en el territorio. \\
\cline { 2 - 2 } & Dificultades en sistema de salud \\
\hline \multirow{2}{*}{ (4) retos para la salud mental indígena. } & Ausencia de información escrita \\
\cline { 2 - 2 } & Ausencia del abordaje de la salud mental en el territorio \\
\cline { 2 - 2 } & Promoción de la salud mental \\
\hline
\end{tabular}

salud mental para los Pueblos Indígenas no existe, eso es más occidente ¿ya? (P1 E1 1:1)

Sin embargo, se ha ido generando una concepción de la misma, a través de los intercambios de saberes con la perspectiva occidental, la cual ha permeado sus prácticas, costumbres y estilo de vida. Como resultado, en la actualidad los pueblos indígenas conciben el concepto de salud mental desde dos perspectivas, una ancestral y otra occidental. Para cada una de estas perspectivas presentamos la concepción que se tiene del término, las prácticas y saberes.

\section{Perspectiva ancestral}

Es entendida desde una perspectiva holística. Se relaciona con dimensiones positivas como la espiritualidad, el equilibrio, la armonía y el buen vivir, a partir de la relación armoniosa del individuo con su familia, la comunidad y la naturaleza.

Hablar de salud mental en comunidad indigena creo que lleva más allá de ese concepto que se maneja desde occidente que hace referencia al bienestar, a emocionalmente y físicamente, ahí si es algo integral (P9 E8 9:5)

que traduce digamos eh, armonía ...ese buen vivir va más allá de lo que uno lo puede entender desde el español, entonces está relacionado a cómo yo me relaciono con la naturaleza, como el... digamos el bosque, el espacio en el que estoy, la monta$\tilde{n} a$ esta... cómo me relaciono con los animales, con el agua, con todo eso y cómo es esa relación con el otro... (P9 E8 9:4).

Es la relación del todo con el cuerpo físico, mental y espiritual es la relación entre el ser, la naturaleza y la madre tierra (P8 E7 8:27).
La salud mental es el estado de armonía de la mente (lo consciente de sí) con las demás expresiones de lo consciente (lo real) del universo, del planeta, de la especie y de las demás especies (P3 E2 3:6).

Por otro lado, los indígenas se refieren a la salud mental como enfermedad, la cual puede originarse no sólo desde lo físico, sino también desde lo emocional y lo espiritual, como producto de la desarmonía entre el territorio, la familia, el medio ambiente y el entorno social, o por la desobediencia a los mayores.

... Entonces al indígena no solamente lo enferma lo físico, sino también lo emocional, el estar mal con el resto de la comunidad ¿sí? (P6 E5 6:12)

es una desarmonización que hay, a veces, con el territorio o en la familia... (P10 E9 10:3).

Entonces, ese concepto de salud mental, para nosotros como amazónicos... no está bien vista la palabra salud mental, o sea no decimos que son locos, sino que decimos que desobedeció a unas órdenes de los mayores (P7 E6 7:6).

\section{Prácticas y saberes ancestrales relacionados con la salud mental}

Uno de los elementos a través de los cuales se aborda la salud mental, son las labores tradicionales para el fortalecimiento de los saberes ancestrales:

Para que haya una buena salud mental tiene usted que haber cumplido todos los trabajos tradicionales... (P5 E4 5:9).

A través de la espiritualidad, se promueve el equilibrio del individuo en relación con los ancestros y el universo. Desde allí, los problemas de 
salud mental se relacionan con el incumplimiento de las leyes ancestrales, ausencia en rituales de protección y armonización:

cuando yo hablo de espiritualidad, muchos de nuestros viejos, de nuestros superiores decían que, si una persona, un niño, nace con un problema de salud mental es porque, ha sido por el comportamiento de esa familia, el comportamiento del papá, de la mamá... de no cumplir las reglas de juego, de no cumplir la Ley de Origen ancestral, del territorio, de ese pueblo, la cosmovisión, la cultura (P8 E7 8:3).

Otro elemento, es la Ley de Origen Natural, donde se tejen relaciones con el entorno:

nosotros actuamos desde las maneras de perspectivas de cosmovisión de origen natural de ese origen primero, ¿verdad?. Como lo es el entorno de nosotros donde convivimos y donde permanentemente estamos haciendo una dinámica de vida con todas las personas que pertenecen a nuestra cultura, a nuestro entorno, a nuestros ambientes ( $\mathrm{P} 4 \mathrm{E} 3$ 4:7).

Además, otros aspectos como la alimentación y las dietas tienen relación con la salud mental:

Para decir que nosotros tenemos un indígena con problemas de salud mental, podemos decir que tuvo un maleficio, o no obedeció a sus dietas, a sus razones estructurales, ancestrales y tradicionales ordenadas por el cacique o por el chamán (P7- E67:5).

Por último, la oralidad es el medio por el cual las comunidades conservan y transmiten el conocimiento propio, incluyendo allí elementos importantes, que difícilmente se encuentran de manera escrita.

Que haya escritos, que esté escrita es difícil, difícil, porque ese es un conocimiento tradicional propio, y los chamanes y los médicos tradicionales que lo han enseñado, lo han enseñado a sus hijos, pero muy poco, no es que esté publicado (P7 E6 7:9).

\section{Perspectiva occidental}

La visión occidental de la salud mental parece ser el resultado de procesos de colonización y aculturación:

\section{Concepción de salud mental}

Para los indígenas de Colombia, la salud mental es un término acuñado por lo occidental, muy nuevo, el cual se relaciona con anormalidad o loquera, dolor de cabeza, confusión y afectación del comportamiento:

Ese concepto de salud mental, o sea lo hablo como amazónico... no pueda existir; porque eso es como una caracterización occidental (P7 E6 7:4).
A la salud mental, loquera es como nosotros le decimos (P1 E1 1:12).

O sea dolor de cabeza eeeh problemas, confusión de cabeza, bueno así se dice, pero salud mental no, no tiene traducción para nosotros (P1 E1 1:14).

esa palabra para los indígenas, que enseguida te la relacionan es con afectación del comportamiento de la persona (P5 E4 5:6).

\section{Prácticas y saberes occidentales}

relacionados con la salud mental

Se explican los problemas de salud mental, como impuestos desde afuera, y el resultante abandono de lo propio:

... Dicen nuestros sabedores, ahora tenemos muchos más problemas, primero, porque estamos interculturalizados; ya lo que somos, lo que éramos nosotros originariamente ya no lo somos; ahora, referente, ya nos interculturalizamos, estamos cediendo a otras cosas, ya no cumplimos... realmente, con gran porcentaje de lo propio de nosotros (P8 E7 8:9).

\section{Elementos de la salud mental}

Los participantes perciben elementos positivos y negativos que influyen en la salud mental. Como factores positivos se encontró que el uso de rituales promueve el vivir bien, la armonía a nivel individual, comunitario y de la madre tierra. Se encontró que, factores como la convivencia, la equidad, la unidad y la participación juegan un papel importante:

...Y por eso es que básicamente ahí se utilizan muchos los rituales y eso, porque el problema de vivir en armonía o vivir sano no es solamente entre... Vivir sano no es solamente estar bien y no tener dolor sino estar bien consigo mismo, con los demás, ¿sí? con los demás miembros de la comunidad y con la madre tierra (P6- E5-6:16).

Se menciona además, que el cumplimiento de las leyes y enseñanzas de los viejos promueven la salud mental, así mismo, la armonía y la convivencia.

Si nosotros todos cumpliríamos las cosas, haríamos caso a las enseñanzas, como a las recomendaciones que hacen nuestros viejos pues estaríamos bien (P8- E7- 8:5).

Para que no hubiera esos problemas que llevan pues actualmente a depresión o a esas cosas, o también peleas en la comunidad que generan pues estrés a nivel mental y/o si una desarmonía (P10E9- 10:12).

La medicina tradicional y la espiritualidad son usadas como medios de curación, y por ello, 
hacen un llamado a que se reconozca la labor de los sabedores tradicionales: médicos, chamanes, sobanderos. Cuando la mente y el cuerpo están conectados se materializa la tranquilidad del espíritu y el equilibrio físico:

... Que se reconozca esa labor que hacen los médicos tradicionales, los chamanes, los sobanderos, las parteras, que se reconozca esa labor, que realmente es una medicina que está beneficiando a las Comunidades Indígenas (P7- E6- 7:11).

Como indigena pienso que la salud debe abordar la mente para que la tranquilidad se manifieste en la comunicación como el mundo energético o del espiritu y el cuerpo que es la expresión material de la tranquilidad en la mente y de la comunicación permanente con el espiritu que se traduce en un equilibrio o desequilibrio físico (P3- E2- 3:9).

Respecto a los elementos negativos, se encontró que las prácticas de origen occidental pueden generar problemáticas, debido al choque cultural, la violencia, la discriminación y otras. Puede ocasionarse por desobediencia a lo ancestral, o incluso perdida de la soberanía alimentaria.

Pero por ejemplo con la cercanía o con el choque cultural con la otra cultura, la cultura occidental, pues ahí vienen problemáticas que, como la discriminación y todo el resto de situaciones que vienen alli, de violencia y otras cosas, y que eso genera cambios, ha generado cambios de comportamiento (P6- E5- 6:3).

.. en general la Ley de víctimas para nosotros la afectación del territorio produce una alteración, por lo cual hablando de Ley de víctimas estamos hablando de la violencia y el estrés que causó y el desplazamiento, entonces eso es parte de la salud mental (P10- E9- 10:16).

Los niños que nacen con problema mental es porque no cumplimos realmente nuestros mandatos, nuestras cosas desde el origen de la parte de origen de cada uno de los pueblos y por eso la tierra, la naturaleza y la madre, madre tierra que, donde existe un Dios supremo, nos castiga por el comportamiento y la irresponsabilidad de nosotros (P8- E7- 8:4).

Por el tema de la alimentación ya nos confundimos, pues en algunos territorios ya no existe el pescado natural, ya no existe, por ejemplo, otras siembras naturales. Ya tenemos problema, y es este tema de la alimentación, dicen nuestros viejos que ha venido afectando a los pueblos indígenas, en este caso, por problemas de salud mental (P8- E78:10).

\section{Escenarios de la salud mental}

En esta categoría se incluyen los avances y ausencias del abordaje de la salud mental en dos escenarios: el territorio y el sistema de salud. Algunos mencionaron avances en sus territorios, que expresan los esfuerzos que se hacen a nivel nacional y local, conformación de grupos técnicos de indígenas, tales como la Asociación de Cabildos Indígenas de Caldas- ACICAL-; establecimiento de rutas y modelos de salud; salud de la mujer, con temas como la ablación y la salud materno infantil; y en la comprensión de la salud mental para lograr estados de armonía y equilibrio:

concomitantemente con este trabajo avanzamos en la conformación de un grupo de apoyo técnico en salud para la ACICAL (Asociación de Cabildos Indígenas de Caldas) (P3 E2 3:3).

En Risaralda, por ejemplo, con el tema de la ablación de Emberá, ellos están trabajando un poco hacia eso, por ese lado de salud mental, pues con mujeres (P6 E5 6:21).

entonces, por lo menos en el momento yo coordino allá el proyecto de salud mental precisamente hemos venido haciendo el ejercicio de decirle a la gente, bueno ¿usted qué entiende por salud mental? Sí, entonces nos hemos encontrado y hecho el trabajo con niños, con sabedores ancestrales y con mujeres (P9 E8 9:15).

A nivel de sistema de salud en el contexto nacional se ha ido consolidando un Sistema Indígena de Salud Propio e Intercultural (SISPI) ${ }^{10}$. En el marco de este esfuerzo nacional se están construyendo los perfiles epidemiológicos municipales y departamentales. Sin embargo, a pesar de los esfuerzos del SISPI, manifiestan algunas limitaciones, como es la no inclusión del tema de salud mental en los programas de salud indígena:

Nosotros estamos construyendo el modelo de salud y lo estamos haciendo en el marco del SISPI, pero vamos en la quinta parte, pero estamos crudos (P6 E5 6:37).

Nosotros tenemos IPS, tenemos EPS, y todavía nos sentimos crudos. Entonces uno se pone a pensar, bueno si nosotros tenemos eso y hemos venido trabajando ya hace ratico, ¿cómo será en Pueblos Indígenas que no tiene nada? (P6 E5 6:39).

Yo me imagino que, por ejemplo, otra de la gente que está muy avanzada son los del CRIC, en el Cauca, ellos hablan del programa de salud del Cauca, aunque yo no los he escuchado hablar sobre ese tema. Ellos han avanzado ya, ellos ya tienen el modelo de salud adelantado, yo me imagino que esa parte, nosotros por ejemplo lo que es salud mental y nutrición estamos crudo en eso, o sea nosotros 
hemos avanzado en otras cosas estamos diseñando por ejemplo proyectos de trabajos con parteras, $u$ programa materno infantil, nutrición con parteras, pero ya la parte de nutrición occidental, la parte de articular eso con lo otro, y hacerle un estudio desde el enfoque occidental no lo hemos hecho ¿sí? En eso estamos crudos y en salud mental también entonces esa es la realidad nuestra, acá... (P6 E5 6:35).

\section{Retos para la salud mental indígena}

Son varios los retos que plantearon los entrevistados, los cuales podrían agruparse en tres puntos, el primero, la importancia de impulsar procesos investigativos acerca de la salud mental de los pueblos indígenas, precisamente por su ausencia:

A nosotros la investigación nos serviría pues para tener herramientas y trabajar una serie de problemáticas que hay; porque en ultimas pues como indígenas, nos interesa abordar esos temas y darles una solución (P6- E5- 6:29).

El segundo reto tiene que ver con la necesidad de avanzar en la consolidación del SISPI, a través del cual se planteen alianzas e intervenciones colectivas entre la academia, el sistema de salud y los pueblos indígenas. Con esto se propone, reconocer la importancia de la labor que realizan los chamanes, sabedores, abuelos, parteras y médicos tradicionales, así como el papel de la medicina tradicional en el buen vivir y mejoramiento del estado de salud de los pueblos indígenas:

... Hacer unas alianzas estratégicas para poder llegar nosotros entre universidades, entre médicos, federaciones de médicos, organizaciones y levantar un verdadero modelo de salud, $y$ tocar todos estos temas y unificar todo lo que llaman el PIC, los famosos proyectos de intervenciones colectivas ( $\mathrm{P} 7-$ E6- 7:18).

Nosotros hemos identificado esa necesidad de avanzar, sobretodo de trabajar con los médicos tradicionales, con parteras y eso, porque ahorita hay muchos problemas (P6- E5- 6:25).

Finalmente, el tercer llamado se relaciona con la necesidad de rescatar y revitalizar el conocimiento oral de los sabedores y de los mayores, mediante la traducción y la escritura del mismo:

... Hablar con los viejos, preguntarle a un viejo directamente, introducirle en la lengua materna de él, porque si usted va y dice: para usted abuelo ¿qué significa para usted la salud mental? De pronto el abuelo es muy corto en castellano, pues nunca te va a dar la respuesta. Pero si de pronto hay un traductor que le diga y se siente, entonces hay una esperanza de que pueda haber un concepto de ese sabedor, de esa sabedora (P8- E7- 8:23).

\section{Discusión}

La aproximación al concepto de salud mental desde la perspectiva de los pueblos indígenas de Colombia constituye un punto de partida para avanzar en la comprensión de su situación, cómo insumo para procesos de promoción de la salud mental, culturalmente apropiada y acorde a dinámicas de interculturalidad entorno a sus significados y prácticas. De este proceso de investigación y ante la pregunta sobre lo que es la salud mental, se obtuvo como respuesta, no solo, sus perspectivas, sino también, los elementos que la constituyen, los escenarios donde actúan dichas prácticas y los retos, lo que permitió tener un panorama más complejo que el inicialmente esperado.

En los resultados algunos participantes manifiestan que la noción de salud mental no existe en el contexto de los pueblos indígenas, lo que visibiliza la necesidad de avanzar en el proceso de construcción intercultural del significado y praxis de ésta.

Como un acercamiento al aporte que hace la cultura indígena colombiana al concepto de salud mental vale la pena resaltar cómo se refiere a estas perturbaciones, como estrés, sufrimiento y aflicción, las mismas que pasan a tener múltiples interpretaciones teóricas: la teoría biomédica ve en estos fenómenos la expresión de una enfermedad orgánica (aunque sea enfermedad mental psiquiátrica), la teoría psicológica los ve como expresión de las emociones y psique individual, y cuando se tiene en cuenta su dimensión social los nombra "psicosocial"18, la teoría sociológica interpreta estos fenómenos como reflejo de los conflictos de clase o de género; pero ninguno de ellos tiene en cuenta el aspecto "moral", que en cambio se hace presente en los hallazgos de la investigación. En su conjunto, las múltiples propuestas insinúan la influencia cultural del grupo social del que provienen dichas apreciaciones.

Los líderes indígenas entrevistados manifiestan diferencias entre el concepto de salud mental indígena y el occidental. El primero, desde su interpretación se refiere a "una manifestación holística e integral que se relaciona con la espiritualidad, el equilibrio, la armonía, el buen vivir, y su relación con la naturaleza, la familia, y la comunidad..."; y el segundo, como resultado de la relación con la cultura no propia u occidental.

En relación a lo anterior y retomando otros conceptos clave, en Colombia la definición expresa en la Política de Salud Mental vigente para el país desde 1998, la cual fue retomada de la de- 
finición de salud mental del Ministerio de salud y bienestar de Canadá (Mental Health for Canadians) afirma que: "salud mental es la capacidad de las personas y de los grupos para interactuar entre sí y con el medio ambiente; un modo de promover el bienestar subjetivo, el desarrollo y uso óptimo de las potencialidades psicológicas, ya sea cognitivas, afectivas o relacionales. Así mismo, el logro de metas individuales y colectivas, en concordancia con la justicia y el bien común"19.

Por su parte, la Ley 1.616 de $2013^{20}$ afirma que "La salud mental se define como un estado dinámico que se expresa en la vida cotidiana a través del comportamiento y la interacción de manera tal que permite a los sujetos individuales y colectivos desplegar sus recursos emocionales, cognitivos y mentales para transitar por la vida cotidiana, para trabajar, para establecer relaciones significativas y para contribuir a la comunidad..."

Como puede verse, aunque estas definiciones incluyen la posibilidad de expresión de la salud mental desde lo individual, lo colectivo, e indican diferentes posiciones en cuanto a ésta, no corresponden directamente a la realidad histórica y cultural del país, y en lo inmediato se diferencian más aún de la concepción de armonía, espiritualidad, buen vivir e integración con la naturaleza y la sociedad.

Sin agotar y solamente insinuar la profundidad en las diferencias, se podría decir que las concepciones obedecen a las lógicas culturales de las que provienen, a los sistemas de pensamiento de cada cultura: la occidental y la de los pueblos indígenas, como la nombran los mismos participantes, en las que cabe aplicar el símil como cultura occidental y cultura oriental. La primera corresponde a un proceso cognitivo analítico y la segunda a uno holístico.

Las características del pensamiento occidental incluyen: la búsqueda de una verdad única, la construcción de contra-argumentos, y la preferencia por la coherencia, mientras la cultura oriental se rige por tres principios: el principio de cambio constante de la realidad; de contradicción como algo constante en coherencia con el cambio; y el holismo o relaciones, esencia del pensamiento dialéctico según el cual nada está solo e independiente, sino que todo está conectado ${ }^{21}$. Otra diferencia se refiere a la percepción del ambiente físico, que en los de occidente tiende a centrarse en objetos sobresalientes en primer plano, mientras que los orientales son más propensos a centrarse en los contextos ${ }^{22}$.

Estas diferencias afectan no solo sus creencias acerca de aspectos específicos del mundo, sino también lo que es importante conocer y cómo se obtiene dicha comprensión, la forma como se dirige la organización social, las prácticas sociales con su influencia directa en el desarrollo, y la definición si se hace uso de procesos cognitivos como la dialéctica o las lógicas ${ }^{23}$. Lo que muestra la trascendencia de los sistemas de pensamiento, inclusive en aspectos de tipo práctico, lo que es claro para el caso de la salud mental, la concepción más o menos integral que se tenga de ella y la conciencia que se tenga o no de las diferencias culturales llevarán a diversos usos de los recursos y a servicios pertinentes o no para cada grupo social.

Podría decirse que a diferencia de otros esfuerzos con mayor trayectoria, que reconocen aspectos culturales de la salud mental ${ }^{24} \mathrm{y}$ los contrastes reflejados en los servicios que se ofrecen ${ }^{25}$, se está dando un lento y tímido proceso de interculturalidad en el caso de Colombia, más aún en contextos donde hay pueblos indígenas; según los hallazgos de investigación y recientes aportes de la academia ${ }^{26,27}$. Adicional a lo anterior, el escaso interés del sector oficial del país se hace evidente, tal como se vio en el caso de 402 integrantes de la comunidad Emberá en condición de desplazamiento en la ciudad de Bogotá, quienes manifestaron con respecto a su salud mental que: "el Estado es el mayor ausente y es quien debe contribuir al desarrollo de intervenciones culturalmente apropiadas" 28,29 .

Es de aclarar que el estudio realizado tiene sus limitaciones en cuanto a la información recolectada, ya que no se pudieron obtener datos de todos los pueblos indígenas del país, lo cual impide que los hallazgos puedan generalizarse $o$ extrapolar las conclusiones a las comunidades no participantes.

A pesar de lo anterior, los aportes en vía a una conceptualización más integral en Colombia, que contemple la diversidad cultural, es un relevante llamado de atención para el país. Valdría la pena continuar un proceso investigativo que evidencie a mayor profundidad y heterogeneidad cultural las concepciones de salud mental y con ellas su aporte al campo y al país, y recomendar de manera más directa aspectos prácticos a tener en cuenta en políticas, planes, programas y proyectos de salud mental en referencia a aspectos culturales.

\section{Conclusiones}

El sistema de salud colombiano ignora las concepciones de las Comunidades Indígenas, por considerar que no es un conocimiento válido, 
por esta razón impone sus intervenciones de manera descontextualizada y homogénea en los Pueblos Indígenas.

Para los Pueblos y Comunidades Indígenas el territorio en toda su dimensión y el cumplimiento a cabalidad de lo ordenado en la Ley de Origen, El Derecho Mayor, La Ley Natural o El Derecho Propio son los pilares fundamentales para mantener el equilibrio y la armonía en la salud, no solo del individuo, sino de la colectividad y todo lo que encierra la Madre Tierra.

Algunas de las acciones que los Pueblos y Comunidades Indígenas proponen son las prácticas de los saberes ancestrales, los diálogos de saberes intergeneracionales y el fortalecimiento de los procesos propios para tener un buen vivir, prevenir el desequilibrio o la enfermedad y mantener la identidad cultural viva en el tiempo para las futuras generaciones.

Ahondar en el concepto de "salud mental" desde la perspectiva de los Pueblos Indígenas implica reconocer que si bien es un constructo no reconocido en su cultura y por tanto creado desde occidente, lo asemejan con otros conceptos propios de su cosmogonía y cosmovisión como lo son la espiritualidad, la armonía y equilibrio entre el entorno social (comunitario, familiar), territorial, espiritual.

Las prácticas tradicionales y los saberes ancestrales son una vía para la promoción de la salud mental en las comunidades, a través de elementos integrales y holísticos como son la Ley de Origen, la ritualidad, la medicina tradicional, la relación con los ancestros, la armonización, las dietas, entre otros.

Los retos que proponen las Comunidades Indígenas frente al tema de salud mental sugieren fortalecer los procesos investigativos desde lo propio, de salud intercultural, y trascender del conocimiento oral de las comunidades al escrito, debido a la permeabilidad de la cultura occidental y el desinterés por la transmisión de saberes ancestrales.

\section{Colaboradores}

EMM Vélez: su aporte estuvo encaminado en la formulación del proyecto de investigación, la recolección de los datos, el análisis e interpretación de la información. Así mismo contribuyó en la dirección y liderazgo de la escritura del artículo y en la revisión crítica tanto del contenido sustancial a plasmarse en el artículo como en la versión a publicarse. JML Ríos: su aporte estuvo encaminado en la formulación del proyecto de investigación, la recolección de los datos, el análisis e interpretación de la información. También contribuyo en la escritura del artículo, en la revisión de la bibliografía que sustentara el mismo y en la revisión crítica de la versión a publicarse. SC Marulanda: su aporte estuvo encaminado en la formulación del proyecto de investigación, el análisis e interpretación de la información. También contribuyó en la escritura del artículo, en la consolidación de la versión final y en la retroalimentación de cada uno de los apartados del artículo. MCV Franco: su aporte estuvo encaminado en el análisis e interpretación de la información. Así mismo contribuyó en la revisión crítica del contenido intelectual sustancial y en la aprobación final de la versión a publicarse. ODM La Rosa: su aporte estuvo encaminado en la formulación del proyecto de investigación, en la búsqueda de actores claves comunitarios indígenas para las entrevistas, el análisis e interpretación de la información desde la perspectiva de las comunidades indígenas. Así mismo contribuyó en la revisión crítica del contenido intelectual sustancial y en la aprobación final de la versión a publicarse. DMH Holguín: su aporte estuvo encaminado en el análisis e interpretación de la información. Así mismo contribuyó en la revisión crítica del contenido intelectual sustancial y en la aprobación final de la versión a publicarse. 


\section{Referencias}

1. Organización Mundial de la Salud (OMS). Salud Mental [Internet]. 2012, [citado 2015 Ago 8]. Disponible en: http://www.who.int/topics/mental_health/es/

2. Tobón FA. La salud mental: una visión acerca de su atención integral. Rev Fac Nac Salud Pública 2005; 23(1):149-161.

3. Hahn R. Anthropology and public health. New York: Oxford University; 2009.

4. Rosique J, Gálvez A, Restrepo MT, Manjarrés LM, Valencia E. Food and Nutrition in Embera Indigenous People. An Ethnogr Glob Landscapes Corridors 2012; 131-156.

5. Aboriginal Medical Services R. NSW Aboriginal Mental Health Report: Dedicated to our children our future. Sydney: NSW Aboriginal Mental Health Report; 1991.

6. United Nations (UN). State of the world's indigenous peoples. Indigenous people's acces to health services. New York: UN; 2010.

7. Organización Panamericana de la Salud (OPAS). Salud en Sudamérica. Panorama de la situación de salud y de las políticas y sistemas de salud. Washington: OPAS 2012.

8. Alto Comisionado de las Naciones Unidas para los Refugiados (ACNUR). Indígenas en las Américas [Internet]. 2009, [citado 2014 Abr 6]. Disponible: http://www.acnur.org/t3/pueblos-indigenas/indigenas-en-las-americas/

9. Inter-Agency Support Group on Indigenous Peoples Issues. The health of indigenous peoples [Internet]. 2014. [citado 2015 Abr 23]. Disponible en: http:// www.un.org/en/ga/president/68/pdf/wcip/IASG Thematic Paper - Health - revl.pdf

10. Subcomisión Nacional de Salud Propia e Intercultural de la Mesa Permanente de Concertación. Líneas de trabajo y prioridades de la Consejería de Medicina Tradicional y Salud Occidental de la ONIC, en busca de alternativas para el buen vivir [Internet]. 2012 [citado 2015 Abr 23]. Disponible en: http://cms.onic.org. co/wp-content/uploads/downloads/2012/02/Doc_SISPI_Unidad_Indigena.pdf

11. Programa de las Naciones Unidas para el Desarrollo (PNUD). Pueblos indígenas. Diálogo entre culturas. Bogotá: PNUD; 2011.

12. Lopera JS, Rojas S. Salud mental en poblaciones indígenas. Una aproximación a la problemática de salud pública. Med UPB 2012; 31(1):42-52.

13. Organización Panamericana de la Salud (OPAS). Estado del arte del conocimiento sobre la salud de los pueblos indígenas de América. [Internet]. 2006 [citado 2015 Nov 3]. Dispobible en: http://www.ops.org.bo/ textocompleto/pi31217.pdf

14. Jürgeh H. Ciencia y técnica como "ideología." 4th ed. Tecnos; 1965. $162 \mathrm{p}$.

15. Patton MQ. Qualitative Research \& Evaluation Methods. Thousand Oaks: Sage Publications; 2001.

16. Organización Nacional Indígena de Colombia. Organizaciones filiales [Internet]. 2016 [citado $2016 \mathrm{Mar}$ 20]. Disponible en: http://www.onic.org.co/onic
17. Strauss A, Corbinn J. Bases de la investigación cualitativa: técnicas y procedimientos para lograr la teoría fundamentada. Medellín: Universidad de Antioquia; 2002.

18. Días LF. A outra saúde: mental, psicossocial, físico-moral. Saúde e doença: um olhar antropológico. Rio de Janeiro: Fiocruz; 1994.

19. Colombia. Ministerio de Salud (MS). Resolución $n^{\circ}$ 2.358, de 1998. Diario Oficial 1998; 13 jul.

20. Congreso de Colombia. Ley no 1.616, de 2013. Colombia; 2013.

21. Peng K, Nisbett R. Culture, dialectics, and reasoning about contradiction. Am Psychol 1999; 54(9):741-754.

22. Miyamoto Y, Nisbett R, Masuda T. Culture and the Physical Environment. Psychol Sci 2006; 17(2):113119.

23. Nisbett R, Peng K, Choi I, Norenzayan A. Culture and Systems of Thought: Holistic Versus Analytic Cognition. Psychol Rev 2001; 108(2):291-310.

24. Almeida N, Ávila MT, Tourinho MF. O conceito de saúde mental. Rev USP 1999; (43):100-125.

25. Department of Health and Human Services (DHHS). Mental Health: Culture, Race, and Ethnicity- A Supplement to Mental Health: A report of the Surgeon General. Rockville: DHHS; 2001.

26. Restrepo-Espinosa MH. Biopolítica: elementos para un análisis crítico sobre la salud mental pública en la Colombia contemporánea. Rev Gerenc y Políticas Salud. 2012; 11(23):39-55.

27. Ruíz LF. La salud mental vista desde la medicina social latinoamericana. Investig Segur Soc Salud 2009; 11:131-142.

28. Vallejo S. Medicina indígena y salud mental. Acta Colombiana de Psicología 2006; 9:39-46.

29. Ruíz LF. Salud mental en tiempos de guerra: Una Reflexión sobre la Relación Conflicto Armado - Salud Mental en el Pueblo Indígena Emberá en Situación de Desplazamiento Forzado, Bogotá 2012 - 2014. Rev Fac Med 2015; 63(3):399-406.

Artigo apresentado em 16/02/2018

Aprovado em 06/08/2018

Versão final apresentada em 08/08/2018 